\title{
NUEVAS HERRAMIENTAS, TECNOLOGÍAS Y TÉCNICAS APLICADAS EN LAS CIENCIAS GEOLÓGICAS: PANORAMA ACTUAL Y PERSPECTIVAS FUTURAS
}

\author{
NEW TOOLS, TECHNOLOGY AND TECHNIQUES APPLIED IN GEOLOGICAL \\ SCIENCES: CURRENT SITUATION AND FUTURE PERSPECTIVES
}

\author{
Andrés Ulloa \\ Grupo Pangeas S.A. \\ Tåsenveien 18D, 0853 Oslo, Noruega \\ grupopangeas@gmail.com
}

(Recibido: 06/06/2014; aceptado: 10/10/2014)

\begin{abstract}
Nowadays the technological advances are revolutionizing the way and efficiency of work and its impact is being reflected in the geological sciences. The new microelectromechanical systems allow the incorporation of a wide variety of sensors in devices such as smartphones and tablets. These instruments are multifunctional and highly portable for data collection and replace others devices in the field as personal computers and PDA. Currently, high precision GPS in conjunction with LIDAR technology and sonar technology are more accessible for geological research. With this technology is possible to generate high-resolution three-dimensional models to complement geological studies. A remote sensing technique like high penetration radar is used for modeling ice thickness and bedrock topography in Antarctic. Modern tridimensional scanning and printing techniques are used in geological research and teaching. Also the advance in computer technology allows handling three-dimensional models on personal computers very efficiently and with different display options.
\end{abstract}

Keywords: Field geology, smartphones, tablets, GPS, LIDAR, sonar, geomicrobiology, space geology, 3D.

RESUMEN: Los avances tecnológicos presentes hoy en día están revolucionando la manera y eficiencia de trabajar, y su impacto se está viendo reflejado en las ciencias geológicas. Los nuevos sistemas microelectromecánicos permiten la incorporación de una gran diversidad de sensores en dispositivos como teléfonos inteligentes y tabletas. Esto los convierte en instrumentos multifuncionales y muy portátiles para la toma de datos en campo en estudios geológicos y sustituyendo otros instrumentos informáticos como computadoras personales y ordenadores de bolsillo. GPS de alta precisión en conjunto con tecnología LIDAR y tecnología sonar son cada vez más accesibles y utilizados para investigaciones geológicas, pudiéndose generar modelos tridimensionales de alta resolución para complementar estudios geológicos. Técnicas de sensores remotos como radar de alta penetración son utilizadas para realizar modelos del espesor 
del hielo y topografía en la Antártida. Modernas técnicas de escaneo e impresión tridimensional son utilizadas tanto en investigación como enseñanza en las ciencias geológicas. Asimismo, el avance en la tecnología informática permite hoy en día manejar modelos tridimensionales en computadoras personales de manera muy eficiente y con diferentes opciones de visualización.

Palabras clave: Geología de campo, teléfonos inteligentes, tabletas, GPS, LIDAR, sonar, geomicrobiología, geología espacial, 3D.

\section{INTRODUCCIÓN}

Al realizar estudios geológicos se requiere observar y colectar datos de afloramientos y/o depósitos inconsolidados para poder tener un entendimiento de los procesos físicos, químicos $\mathrm{y}$ biológicos que han ocurrido a lo largo del tiempo geológico (Coe, 2010a). Aunque muchos métodos analíticos y técnicas utilizadas para complementar estudios han evolucionado enormemente, la mayoría de las técnicas utilizadas en la geología de campo no han cambiado en muchos años de desarrollo de la ciencia. Inclusive a inicios de este milenio las herramientas de campo más comunes continuaban siendo el mapa, la libreta, la brújula, piqueta y lupa.

Actualmente existen diversos dispositivos electrónicos que permiten tomar datos geológicos de campo y almacenarlos de una manera muy eficiente. Entre estos instrumentos se encuentran las computadoras portátiles, ordenadores de bolsillo o PDA (personal digital assistant), tabletas y teléfonos inteligentes. Tanto las tabletas como los teléfonos inteligentes resultan muy convenientes para la toma de datos científicos por la diversidad de sensores que presentan, la portabilidad, autonomía y posibilidad de instalar aplicaciones específicas.

Algunas de las técnicas topográficas como LIDAR y batimetría sonar también son más comunes y más accesibles actualmente. En la actualidad, estas técnicas se integran principalmente en estudios geológicos de diversas índoles, tales como deformaciones neotectónicas, análisis de deslizamientos, cálculos de tasas de sedimenta- ción, entre otros. Gracias a la constante mejora en la tecnología aplicada a la computación, las computadoras personales tienen la capacidad de procesar y manejar datos y modelos 3D. Hace algunos años estos modelos eran más exclusivos para las grandes industrias (minería y petróleo por ejemplo) y pocos geólogos manejaban este tipo de herramientas, pero hoy en día, si se quiere estar a la vanguardia, los geólogos deben aprender a manejar este tipo de modelado.

El desarrollo tecnológico que se utiliza hoy en día para las investigaciones geológicas fue la tecnología de punta de hace algunas décadas. Actualmente el Curiosity explora la superficie de marte en el cráter Gale y se encuentra equipado con una gran diversidad de instrumentos analíticos como cromatografos de gases, difractómetro de rayos $\mathrm{x}$, espectrómetro de rayos $\mathrm{x}$, cámaras, espectrómetro de descomposición inducida por láser, espectrómetro de neutrones, entre otros. Todos estos instrumentos tienen como objetivo realizar mediciones in situ de rocas y minerales, suelo y atmósfera (Grotzinger et al., 2012). Por lo tanto no es de extrañar que en algunas décadas existan instrumentos portátiles para análisis geoquímicos y mineralógicos.

En esta revisión se mencionan algunas de las herramientas tecnológicas más útiles que están siendo empleadas cada vez más en el área de la geología. Además, se mencionan algunas posibles metodologías de trabajo que podrían ser de práctica común en el futuro cercano, o lo son en algunas instituciones. Por otra parte, se mencionan algunas de las nuevas áreas de la 
geología que han surgido en décadas recientes para generar un panorama amplio de hacia dónde se podrían dirigir las investigaciones geológicas en los siguientes años.

\section{GEOLOGIA DE CAMPO: PRESENTE Y FUTURO}

En la geología de campo la observación es el rol primordial que debe realizar todo geólogo. Las mismas son apoyadas por imágenes y la toma de muestras. La interpretación de estos elementos son utilizados para realizar un modelo geológico. Este rol no ha cambiado en varios cientos de años, pues la observación es parte fundamental de las ciencias geológicas. El cambio que actualmente está ocurriendo es la manera en que se colectan y procesan los datos de campo.

A continuación se presentan algunas de las herramientas tecnológicas más utilizadas para la toma de datos de campo. Para efectos de este artículo, se mencionan computadoras portátiles, PDA, teléfonos inteligentes y tabletas. En muchas ocasiones, la diferencia entre estas categorías no es muy clara. Algunas casas comerciales que combinan características de una tecnología con la otra haciendo una especie de "híbrido" para ofrecer un nuevo producto en el mercado. Los sistemas operativos que utilizan estos equipos también varían de acuerdo a las casas comerciales, algunos de los más comunes son: Android, iOS, Windows Phone, Blackberry OS, Linux, Windows, entre otros. La diferencia entre una computadora portátil y una tableta, principalmente es el diseño, capacidad de procesamiento y que estas últimas tienen la opción de usar tarjetas SIM. Las tabletas y teléfonos inteligentes tienen funciones muy similares, no obstante, las tabletas por lo general tienen pantallas superiores a 7 pulgadas, lo que las hacen más cómodas para la visualización de datos en el campo. Los ordenadores de bolsillo (PDA) fueron predecesores de los teléfonos inteligentes, aunque tiene funciones similares, por lo general no incorpora tantos sensores como los teléfonos inteligentes.

\section{Computadoras portátiles y ordenadores de bolsillo}

\section{Computadoras portátiles}

Las primeras computadoras portátiles fueron desarrolladas a partir de 1981 primordialmente con fines científicos y militares. Para el año 1995, las ventas de computadoras portátiles se incrementaron notablemente y para el tercer trimestre del 2008 las ventas de portátiles superaron a los ordenadores de escritorio (Scheck \& Chao, 2009). En algunas áreas de la geología como la geofísica, el uso de las computadoras portátiles es muy común; sin embargo, su uso no es muy popular para la geología de campo.

Las ventajas de las computadoras portátiles en el trabajo de campo son muchas, pues por lo general se puede pasar la información de una vez en el formato deseado o utilizar la mayoría de softwares requeridos. No obstante, estas solo aplican cuando los sitios de estudio son muy accesibles o se realiza mapeo geológico en afloramientos con acceso en carro y en este se puede tener la computadora. Las desventajas es que por lo general no son tan compactas para transportarlas, no son muy resistentes a las condiciones ambientales difíciles y generalmente no poseen GPS ni cámara de alta calidad. Existen algunas marcas comerciales que ofrecen modelos robustecidos muy útiles para el trabajo de campo resistente a caídas, agua y polvo. No obstante, su precio es bastante más elevado que el de una computadora portátil tradicional.

\section{Ordenadores de bolsillo}

El primer modelo comercial de ordenadores de bolsillo apareció en 1992 (Apple Newton) y fueron populares en la década de los noventas e inicios del nuevo milenio (Berkan, 2012). Estos tienen la ventaja de contar, por lo general, con un sistema operativo compatible con computadoras, por lo que algunos de los programas que se instalan en estas también pueden ser instalados en 
estos dispositivos. Actualmente están siendo remplazados por los teléfonos inteligentes, por lo tanto cada vez son menos frecuentes en el mercado.

Algunos modelos con sistema operativo de Windows permiten instalar algunos programas en los que se pueden colectar los datos de una vez en el campo. Esto incluye algunos SIG (Sistemas de Información Geográfica), software tipo CAD para mediciones topográficas, bases de datos, entre otros. También existen modelos robustos, y uso de tarjetas SIM que permiten enviar datos vía internet, así como la conexión bluetooth permite trabajar conjuntamente con otros instrumentos como equipos topográficos.

\section{Teléfonos inteligentes y tabletas}

A partir del 2007, la pantalla multitáctil del iPhone lleva a los teléfonos a nuevas alturas, permitiendo que a partir de los teléfonos celulares se pudiera hacer llamadas, mensajear, jugar, tomar fotos y navegar en Internet. Para el 2008, Google lanza el sistema operativo Android y rápidamente se volvió el sistema operativo dominante en los teléfonos inteligentes (Berkman, 2012). El avance tecnológico en los sistemas microelectromecánicos (MEMS Microelectromechanical Systems) ha permitido la incorporación de gran cantidad de sensores en los teléfonos inteligentes. De acurdo con Torres-Salinas (2011), las opciones que presentan estos aparatos como conectividad $3 \mathrm{G}$, Wifi, códigos QR, capacidad de registro (fotos, video, voz, entre otros), los convierten en verdaderos laboratorios móviles, por lo que su impacto en la investigación científica está empezando a ser evidente y a extenderse. En el caso de la geología los alcances de los teléfonos inteligentes como tabletas ya están mucho más allá de solamente medir buzamientos y georreferenciación de datos.

En el cuadro 1 se encuentran algunos de los sistemas microelectromecánicos y funcionalidades más comunes incorporados en los teléfonos inteligentes y tabletas. Se describen sus principales características y posibles aplicaciones en las ciencias geológicas (Fig. 1).
Como un ejemplo particular de este tipo de aplicaciones, Ndiaye \& Diagne (2014) demostraron que a partir de mediciones de campo magnético se puede delimitar algunos cuerpos ígneos. El experimento se realizó en Senegal, oeste de África, donde utilizaron un iPhone y una persona caminando sobre un terreno. En este sito la geología presenta un alto contraste de campo magnético entre rocas sedimentarias e intrusivos basálticos, integrando datos de localización y campo magnético muestreados con una frecuencia de $30 \mathrm{~Hz}$. A partir del estudio determinaron que es posible diferenciar litologías con campos magnéticos contrastantes con el uso de un teléfono inteligente.

Otra aplicación que podría ser de utilidad en las ciencias geológicas es para medir contaminación de polvo fino. En el Instituto de Tecnología de Karlsruhe de Alemania se trabaja en un prototipo que se puede conectar a los teléfonos inteligentes para elaborar mapas de contaminación. El principio de funcionamiento es la luz emitida por el led del teléfono que es dispersada por el polvo o humo que pueda existir. La cámara sirve como un receptor y toma una imagen donde el brillo de los pixeles se puede correlacionar con la contaminación por polvo Gómez (2014).

Aplicaciones orientadas a respuesta temprana ante amenaza sísmica están siendo también investigadas. Los acelerómetros presentes en los teléfonos inteligentes pueden registrar de manera confiable los sismos y podrían desarrollarse sistemas de detección temprana utilizando la red de teléfonos celulares (Faulkner et al., 2011). De acuerdo con D'Alessandro, \& D'Anna, (2013) los acelerómetros MEMS son adecuados para adquisición sísmica de eventos fuertes. Esto podría incrementar considerablemente el número de datos sísmicos a la hora de que ocurre un sismo. Por lo tanto, un dispositivo con sensores MEMS conectado a internet podría convertirse en una potencial estación sismológica.

Weng et al. (2012) indican que la aplicación en plataforma Android GeoTools puede ser utilizada para múltiples funciones del trabajo de campo y con esta es posible registrar datos de bu- 
Cuadro 1

Sistemas microelectromecánicosnicos y funcionalidades más comunes incorporados en los teléfonos inteligentes y tabletas

\begin{tabular}{|c|c|}
\hline MEMS y funciones & Posibles aplicaciones geológicas \\
\hline Celular & Envío de la información vía internet para realizar respaldo de datos \\
\hline Cámara & $\begin{array}{l}\text { Registro fotográfico de afloramientos y geomorfología. Medición de difracción } \\
\text { de la luz para aplicaciones específicas }\end{array}$ \\
\hline \multirow[t]{2}{*}{ Micrófono } & Registros de audio \\
\hline & Medición de decibeles para aplicaciones ambientales \\
\hline Pantalla táctil & Ingreso de información de manera práctica, anotaciones y dibujos de campo \\
\hline GPS & Ubicación geográfica (Algunos tienen precisiones de 3m) \\
\hline Acelerómetro & $\begin{array}{c}\text { Acelerómetro en } 3 \text { ejes que permite cumplir funciones de geófono para colecta } \\
\text { de información geofísica y sismológica }\end{array}$ \\
\hline Giroscopio & $\begin{array}{l}\text { En combinación con el acelerómetro puede brindar información de aceleración } \\
\text { y rotación angular en } 3 \text { ejes }\end{array}$ \\
\hline \multirow[t]{2}{*}{ Magnetómetro } & $\begin{array}{c}\text { Como brújula. Combinado con el acelerómetro puede medir la orientación } \\
\text { tridimensional }\end{array}$ \\
\hline & $\begin{array}{l}\text { Posible prospección de yacimientos metálicos que pueden producir variaciones } \\
\text { considerables del campo magnético }\end{array}$ \\
\hline Luxometro & $\begin{array}{c}\text { Pueden ser utilizados en geología ambiental para evaluar condiciones de } \\
\text { iluminación }\end{array}$ \\
\hline Sensor de presión & $\begin{array}{c}\text { El sensor de variación de presión barométrica puede utilizarse en asistencia } \\
\text { con el GPS para determinación de la altura }\end{array}$ \\
\hline Sensor de temperatura & Algunas mediciones de temperatura para geología ambiental \\
\hline Bluetooth & $\begin{array}{c}\text { Transferencia de datos y conexión a distintos instrumentos como estaciones } \\
\text { topográficas o sensores externos }\end{array}$ \\
\hline
\end{tabular}

zamientos. La aplicación también integra registro fotográfico, de video, de audio, notas, coordenadas GPS y puede exportar los datos a formato XML. Una aplicación en plataforma Android muy popular y recomendable para el manejo de bases de datos de campo es Memento Database LuckyDroid@ 2014. Esta aplicación permite una gran flexibilidad en el tipo de campos a registrar aprovechando las herramientas del dispositivo. Además permite la captura de datos de una manera sistemática y ordenada y puede integrarse con google drive para poder subir los datos a una plantilla compartida en la web. Existe gran diversidad de aplicaciones que pueden instalarse en los teléfonos inteligentes y tabletas, las cuales responden a diferentes necesidades científicas, algunas son de libre acceso y otras es necesario pagar por la instalación.

\section{SISTEMAS DE POSICIONAMIENTO GEOGRÁFICO (GPS) Y TOPOGRAFÍA}

Una herramienta fundamental para el geólogo son los mapas topográficos pues es la referencia del terreno donde se colocan las observaciones de campo. Algunos de los geólogos pioneros del siglo XIX como William Gabb, tuvieron primero que crear mapas cartográficos para luego poder hacer su modelo geológico (Denyer \& Soto, 1999). Actualmente, se puede conseguir información topográfica digital en diferentes formatos y escalas, y el uso de los SIG facilita mucho el manejo de los datos. Hoy en día se encuentran modelos de elevación de prácticamente todo el planeta, inclusive la batimetría. Muchos de estos son de libre acceso $\mathrm{y}$ poseen suficiente resolución para trabajos 


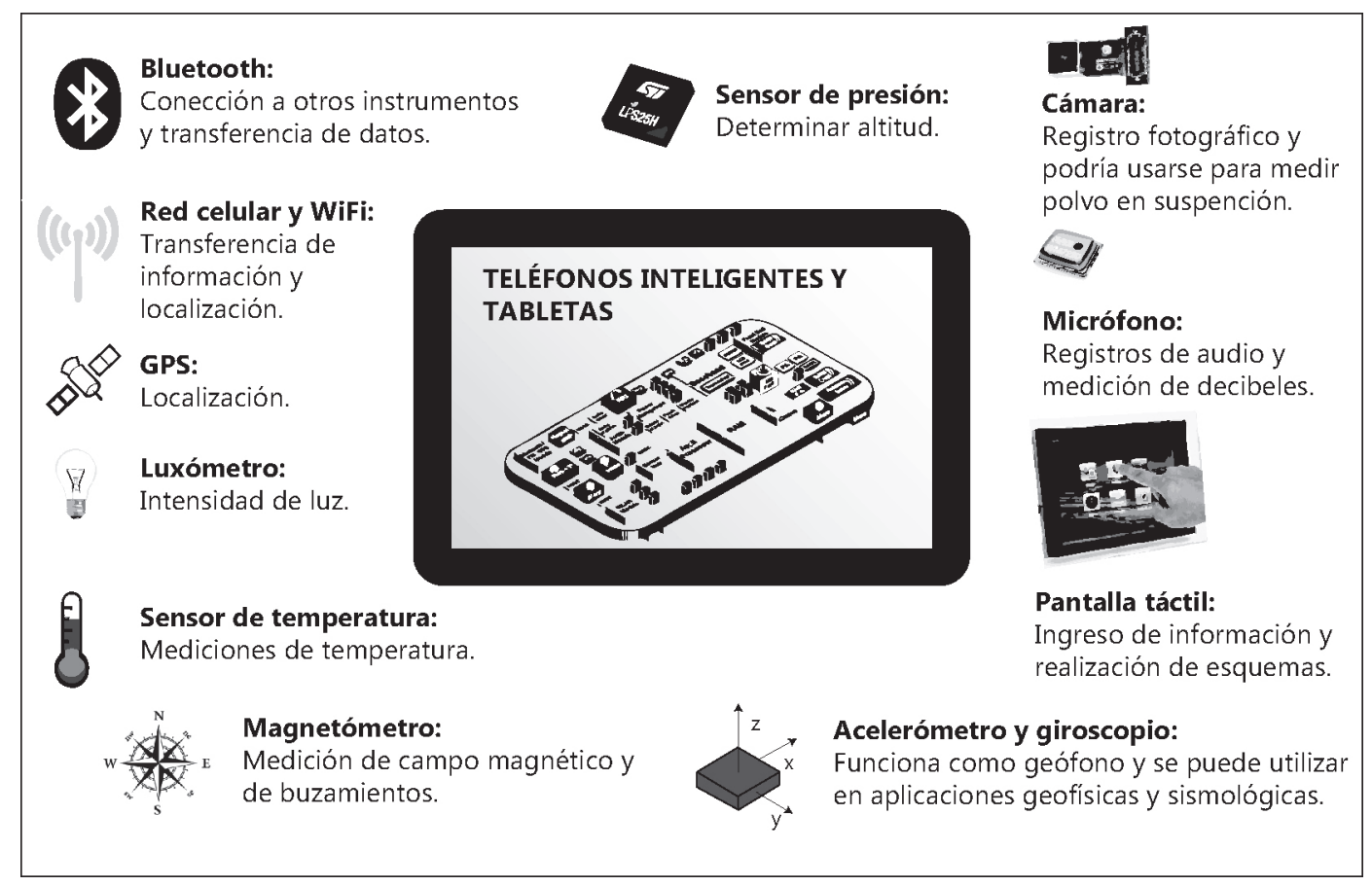

Fig. 1: Esquema de posibles usos y aplicaciones para ciencias geológicas de los dispositivos presentes en teléfonos inteligentes y tabletas.

geológicos regionales. Existen opciones con las que inclusive es posible obtener resolución topográfica milimétrica.

\section{Sistemas de posicionamiento geográfico (GPS)}

Los receptores GPS son dispositivos que a partir de ultra-altas frecuencias de radio de satélites son capaces de triangular la posición con algunos pocos metros de error lateralmente, con menor precisión en la altitud (Coe, 2010b). La mayoría de geólogos de la época estamos familiarizados con esta tecnología, pues desde 1995 la señal GPS es brindada a la comunidad civil, y con ello una gran serie de dispositivos se encuentran en el mercado. En el caso de las ciencias geológicas, el GPS se convirtió en una herramienta indispensable para el trabajo de campo. Los dispositivos convencionales brindan precisiones de hasta $3 \mathrm{~m}$ de error. Equipos mucho más precisos capaces de realizar mediciones milimétricas son también utilizados para algunos trabajos como deformaciones neotectónicas, pero tienen un elevado precio por su alta especificidad.

\section{Tecnología LIDAR}

La tecnología LIDAR (Light Distance and Ranging), fotogrametría y otros sensores han presentado una rápida evolución debido a su aplicabilidad en las industrias en topografía y mapeo. Actualmente esta tecnología ha llegado a ser suficientemente reducida en términos de tamaño y peso para ser considerada por plataformas UAV (Unmanned Aerial Vehicle) (Fig. $2 b$ ). Hay equipos ligeros y portátiles capaces de generar 700000 puntos de distancia por segundo con un ángulo de visión de $40^{\circ} \mathrm{y}$ cabezas rotatorias que pueden girar más de 20 veces por segundo y proveer información con precisión menor a $2 \mathrm{~cm}$ (GIM INTERNATIONAL, 2013; 2014).

Los modelos tridimensionales a partir de imágenes LIDAR permiten representar de manera muy exacta superficies muy irregulares como cavernas. En la Cueva de los Cristales situada en la mina NAICA, Chihuahua México, se realizó en el 2007 un escaneo laser con LIDAR para tener un modelo 3D de la caverna con los 
cristales de yeso más grandes conocidos hasta el momento, que alcanzan hasta $11 \mathrm{~m}$ de longitud. Dicha medición se realizó en un ambiente extremo de 50 grados centígrados a humedad cercana al 100\% (Canevese et al., 2009). Este modelo tridimensional de alta resolución permitió realizar observaciones geológicas basadas en los modelos computacionales. Otro ejemplo de un modelo tridimensional para una cueva se realizó en el karst del sur de China, en la cueva Miao Room's (National Geographic, 2014).

Con base en modelos tridimensionales generados a partir de LIDAR, se puede también obtener información geomecánica para excavaciones subterráneas con la ventaja de evitar atrasos en el avance de la obra. Con estos modelos se pueden realizar caracterizaciones geomecánicas de las discontinuidades como inclinación, localización, orientación frecuencia y rugosidad (Fekete et al., 2010). La tecnología LIDAR también ha sido utilizada como una herramienta de mapeo geomecánico muy efectiva en la industria minera (Lato et al., 2010), en el monitoreo de amenazas por caída de rocas (Lato et al., 2009, 2014) o modelado de inundaciones (Mason et al., 2004). Lato et al (2014) mencionan que el uso de sensores remotos en el mapeo y monitoreo de terrenos montañosos a través de sensores remotos (LIDAR y fotogrametría) es posible mapear cambios topográficos con una gran precisión (Fig. 2).

\section{Sonares}

El mapeo bajo el agua no es un problema. La tecnología SONAR también permite realizar estudios batimétricos muy útiles para estudios de lagos o sistemas kársticos subterráneos. Aunque es una tecnología relativamente antigua, recientemente existen dispositivos de sonar compactos y portátiles, muy útiles para aplicaciones geológicas. Aunque la tecnología sonar no es tan rápida y precisa como los escaneos laser, robots como el modelo DEPTHX desarrollado por Stone Aerospace realiza modelos $3 \mathrm{D}$ de con precisiones de $10 \mathrm{~cm}$, como el modelo del sistema Zacatón en México (Gary et al., 2008). Otras posibles aplicaciones son la batimetría de lagos, ríos, esteros y mares, estimación de tasas de sedimentación, entre otros.

Hoy en día, es posible realizar modelos topográficos inclusive bajo capas de hielo, como lo realizó el equipo de trabajo de British Antartic Survey, en la Antártida. A partir de décadas de toma de datos geofísicos así como medidas de elevación de la superficie tanto aérea como satelitales y el uso de radares de alta penetración se generó un modelo topográfico bajo la superficie de hielo de la Antártica. Dicho modelo es el Bedmap2 y podrá ser utilizado para modelado de las capas de hielo, oceanografía física y aplicaciones geológicas (NASA, 2013).

\section{MÁS DE DOS DIMENSIONES}

Tradicionalmente las representaciones geológicas se suelen trabajar en dos dimensiones, ya sea una planta (mapas geológicos) o perfil (perfiles geológicos). Este método es una forma de representar cuerpos geológicos en 3D descomponiéndolos en imágenes perpendiculares $2 \mathrm{D}$, que ha sido muy efectivo a lo largo de los años. Las representaciones $3 \mathrm{D}$ de cuerpos han sido muy utilizadas en la industria petrolera y minera en las últimas décadas, y en general estos procesos se habían caracterizado por ser costosos y muy especializados. La creciente mejora de la capacidad de procesamiento de los ordenadores y softwares de modelado $3 \mathrm{D}$ ha permitido que ahora sea posible desarrollar modelos geológicos 3D en computadoras personales.

Algunos programas como AutoCAD, Surfer, Voxler y ArcGIS son comunes en el área de la geología y permiten hacer representaciones tridimensionales de superficies o cuerpos geológicos. También existen muchos programas libres para modelados 3D que pueden ser utilizados para distintos fines. A partir de datos de alturas conocidos, ya sean datos puntuales o poli-líneas con valores de alturas, se pueden generar superficies que correspondan al terreno, contactos geológicos, contactos geofísicos, niveles freáticos, entre otros, a partir de puntos o líneas. De esta manera es posible modelar en $3 \mathrm{D}$ diferentes volúmenes (Fig. 3). 


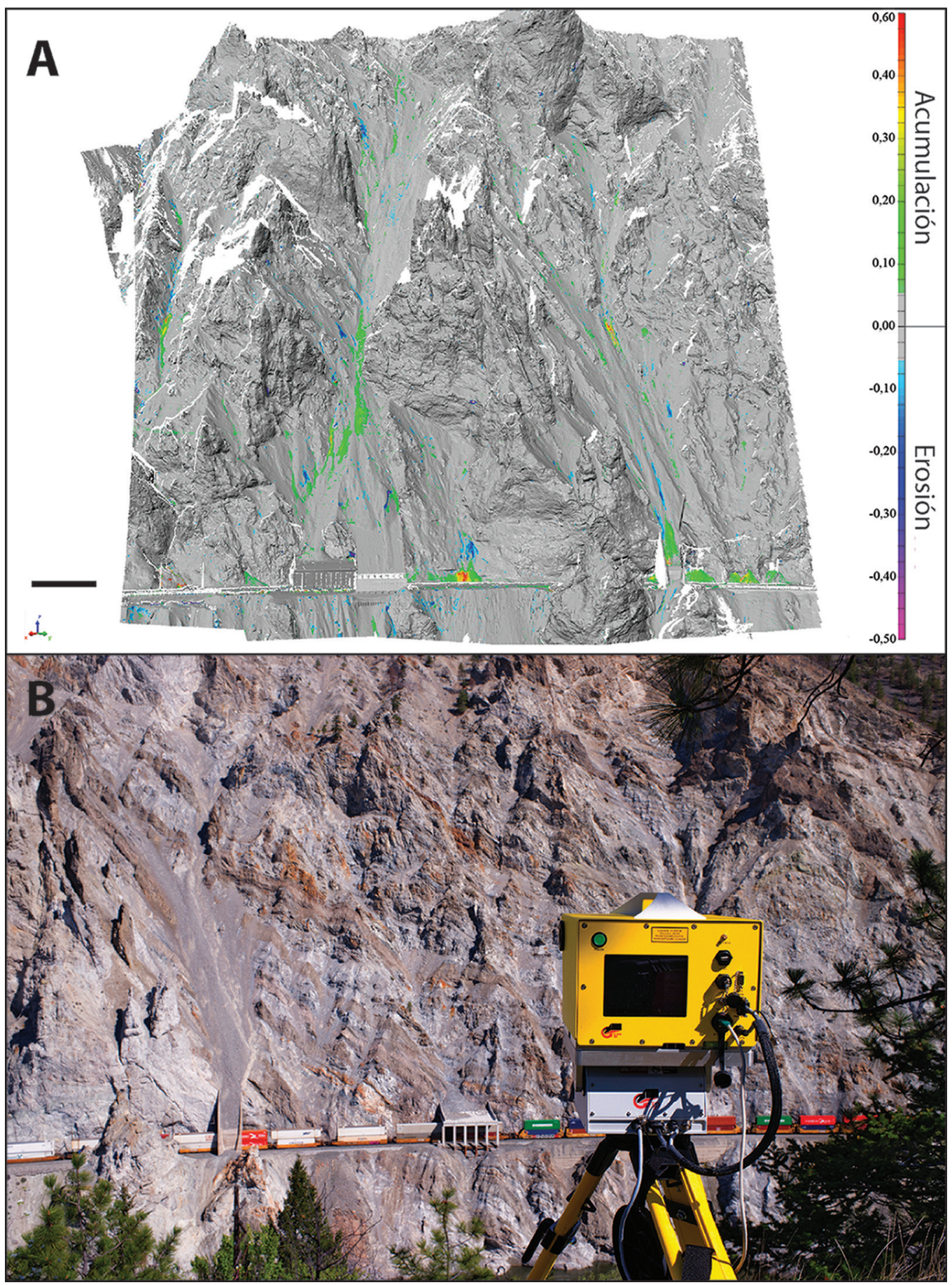

Fig. 2: Ejemplo de utilización de tecnología LIDAR para el monitoreo de caída de rocas en una vía férrea en la Columbia Británica, Canadá. En dicho estudio se colectaron datos del macizo rocoso en numerosas ocasiones para analizar donde ocurren las caídas de roca y que tan frecuentes son. A) Modelo de acumulación y erosión del macizo rocoso obtenido para un periodo de 5 meses. B) Fotografía del sitio y el equipo utilizado. Imagen y fotografía cortesía de Matthew J. Lato (RockScense GeoSolutions Inc). 
Un poco más complicado es el modelado de cuevas en sistemas kársticos, donde se requiere levantar una poligonal (contemplando alturas y anchos) para modelar la cueva. El modelado se puede realizar en programas de cómputo gratuitos como Compass, XTerion, Visual Topo, Loch, entre otros. Estos modelos de cuevas, pueden exportarse a escenas 3D donde se puede incluir la superficie del terreno.

Tradicionalmente la manera en que los geólogos visualizamos la tercera dimensión es a través de la fotointerpretación con estereoscopio. Esta técnica a pesar de ser muy efectiva, tiene la desventaja de que las fotografías con las interpretaciones deben ser georreferenciadas posteriormente para la elaboración de mapas. Esto se puede solucionar con el uso de estereoscopios para computadoras en conjunto con fotografías aéreas georreferenciadas. Otra manera de visualizar la tercera dimensión es con el uso de anáglifos, que son imágenes en dos dimensiones capaces de generar un efecto tridimensional cuando se ven con lentes especiales (Fig. 4). Este método puede ser utilizado para fotointerpretación de fotos aéreas, darles un mayor efecto de profundidad a modelos tridimensionales o crear el efecto de profundidad en fotos ordinarias.

Actualmente en la industria petrolera existen salas de visualización que utilizan técnicas de modelado 3D para analizar los reservorios de petróleo. Una técnica que podría implementarse en el futuro cercano, que hace algunos años podía ser considerada "ciencia ficción" es el uso de hologramas para representaciones geológicas tridimensionales. Los hologramas son técnicas fotográficas avanzadas que permiten generar imágenes en tres dimensiones. Actualmente empresas como Zebra Imaging venden el servicio de hologramas para aplicaciones científicas, arquitectónicas y militares.

Otra técnica innovadora que podrían revolucionar la manera en que se hace investigación y se enseñan las ciencias geológicas es el escaneo e impresión en tres dimensiones. Estas técnicas es posible imprimir fósiles, patrones de fracturas, rocas porosas, minerales, etc. También permitiría experimentar con espacios porosos, imprimiendo diferentes modelos (Oskin, 2013).

\section{DISCUSIÓN Y CONCLUSIONES}

A partir de los años 90's se ha dado una importante evolución en la tecnología y el uso de tecnologías informáticas ha sido cada vez más común desde entonces Aunque la manera tradicional en la que se realiza geología se ha mantenido relativamente constante en el uso de la libreta y mapa de campo, brújula, GPS (recientemente incorporado), dibujos descriptivos y fotografías, la tecnología actual permite integrar la mayoría de instrumentos en un solo dispositivo como un teléfono inteligente o tableta.

Actualmente por el mismo precio, alrededor de $\$ 500$ se puede comprar una brújula geológica (Ejemplo: Brunton Geo Pocket) se puede comprar una Tableta (ejemplo: Sony Xperia). La pequeña diferencia es que si se compra la brújula, solo se puede tomar datos de buzamientos y hay que apuntarlos en la libreta. Por otro lado, si se elige la Tablet, puede tomar y grabar datos de buzamientos, añadirles atributos, georreferenciarlos, tomar fotografías de calidad del afloramiento, tener mapas del sitio, enviar los datos vía internet y un sinfín de aplicaciones mencionadas en este artículo. ¿Cuál elegiría usted? El reto de los geólogos actuales es poder adaptarse a estos cambios tecnológicos presentes, para poder aprovechar al máximo los recursos tecnológicos. Esto implica establecer metodologías de trabajo con protocolos de toma de datos automatizados y el manejo adecuado de las tres dimensiones.

Hoy en día existen diferentes mecanismos como LIDAR para la obtención de modelos topográficos suficientemente detallados que permiten realizar mediciones hasta caracterizaciones geológicas de fracturas a partir de los modelos generados (Fekete et al., 2010). Con esta tecnología se puede realizar modelos tridimensionales de muy alto detalle de superficies irregulares complejas como cuevas (Canevese et al., 2009). Bajo el agua es posible utilizar tecnología sonar para obtener modelos batimétricos con resoluciones de hasta $10 \mathrm{~cm}$ (Gary et al., 2008). Combinando técnicas topográficas convencionales con geofísica y el uso de tecnología radar de alta penetración en la Antártida 


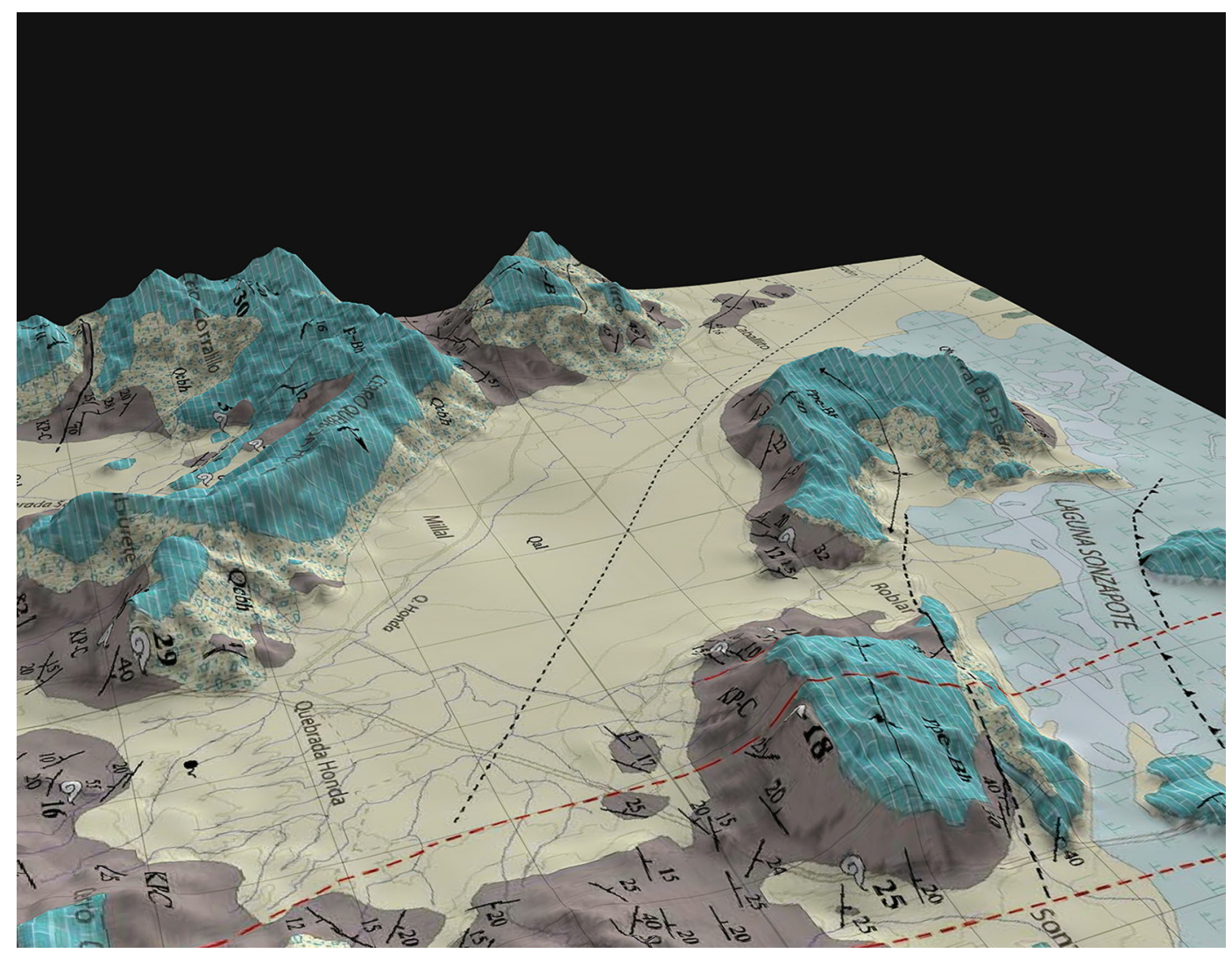

Fig. 3. Modelo geológico tridimensional de parte de la hoja Talolinga. Modelo de elevación generado a partir de las curvas de nivel cada 10 metros del proyecto TERRA. Mapa geológico tomado de Denyer et al. (2013).

fue posible determinar los espesores de las capas de hielo y un modelo de la superficie bajo esta (NASA, 2013).

A partir de datos tridimensionales (coordenadas y elevación), en una computadora personal y con los softwares adecuados es posible realizar modelos tridimensionales complejos de terrenos. Esta tipo de modelado se utiliza con mayor frecuencia, pues permite representaciones más realísticas que los tradicionales planos y perfiles. Algunas herramientas de visualización como anáglifos, pantallas 3D u hologramas ayudan a crear modelos más realísticos. El manejo adecuado de las tres dimensiones actualmente es una herramienta muy útil para las investigaciones geológicas y en el futuro cercano se va a ir incrementando y posiblemente va a ser indispensable.

\section{AGRADECIMIENTOS}

Un especial agradecimiento a Percy Denyer, quien me motivó de escribir este artículo y por sus sugerencias. También se le agradece por brindar la base geológica cartográfica para la figura 3. A Matthew J. Lato de RockScense GeoSolutions Inc. por facilitar la imagen y fotografía de la figura 2 y las explicaciones correspondientes.

\section{REFERENCIAS BIBLIOGRÁFICAS}

BERKMAN F., 2012: How the Cellphone Got 'Smart'. Mashable.- http://mashable. com/2012/10/15/cellphones-smartphones/ [Consulta: 26 de Junio de 2014]. 


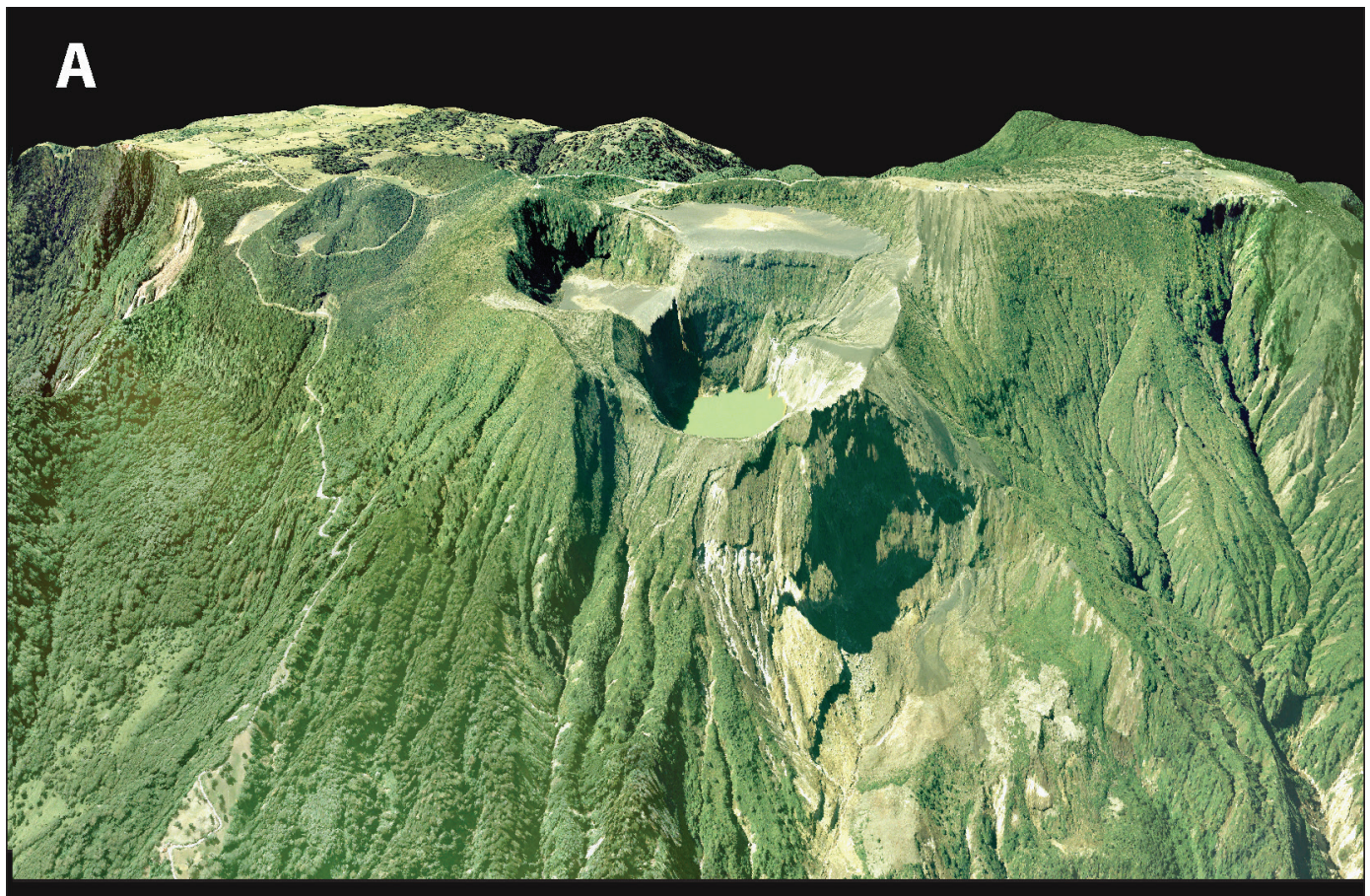

$\mathbf{B}$

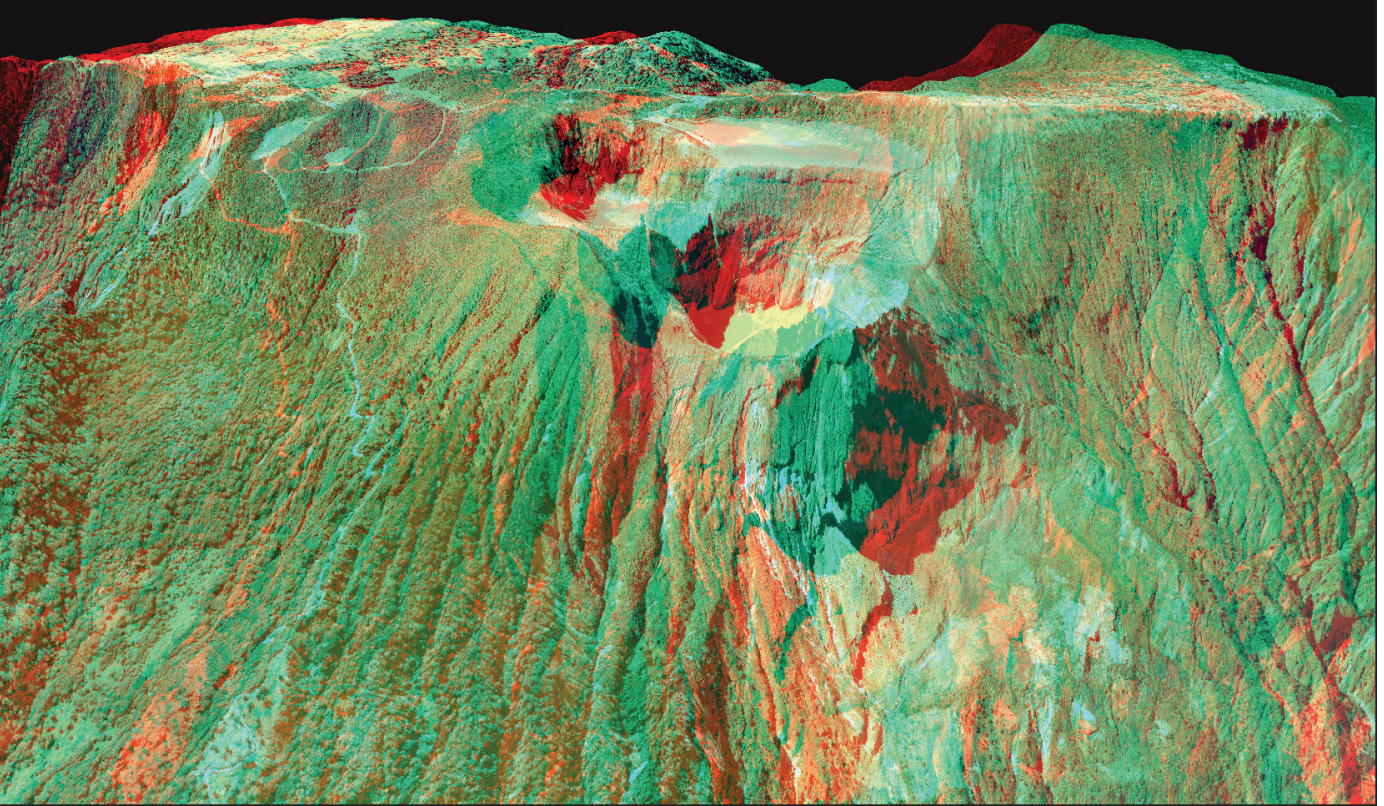

Fig.4: Modelo tridimensional del volcán Irazú. A) Vista en perspectiva del modelo. B) Vista para anáglifos. Modelo de elevación generado a partir de las curvas de nivel cada 10 metros del proyecto TERRA. Fotografías aéreas obtenidas de Catastro Nacional, año 2005. 
CANEVESE, E.R., TEDESCHI, R. \& FORTI., P., 2009: The caves of Naica: laser scanning in the extreme underground environments.The American Surveyor. -http://www.amerisurv.com/PDF/TheAmericanSurveyor CavesOfNaica_February2009.pdf html [Consulta: 26 de Junio de 2014].

COE, A. (Ed.), 2010a: Geological field techniques.- 323 págs. Blackwell Publishing Ltd, London, UK.

COE, A., 2010b: Field equipment and safety.En: COE, A. (ed.): Geological field techniques.- Blackwell Publishing Ltd., London, UK: 4-41.

D'ALESSANDRO, A. \& D'ANNA, G., 2013: Suitability of low-cost three-axis MEMS accelerometers in strong-motion seismology: Tests on the LIS331DLH (iPhone) accelerometer.- Bull. Seismol. Soc. Amer. 103(5): 2906-2913.

DENYER, P. \& SOTO, G., 1999: Contribución pionera de William M. Gabb a la geología y cartografía de Costa Rica.- Anuario de Estudios Centroamer. 25(2): 103-138.

DENYER, P., AGUILAR, T. \& MONTERO, W., 2013: Mapa geológico de la hoja Talolinga.Escala 1:50 000, Ed. Universidad de Costa Rica, San José.

DORRIER, J., 2013: Sensors in smartphones: Galaxy S4 adds pressure, temperature and humidity sensors. SinngularityHUB.http://singularityhub.com/2013/04/01/sensors-in-smartphones-galaxy-s4-adds-pressure-temperature-and-humidity-sensors/ [Consulta: 01 de Julio de 2014].
FAULKNER, M., OLSON, M., CHANDY, R., KRAUSE, J., CHANDY, K. M., \& KRAUSE, A. (2011): The next big one: Detecting earthquakes and other rare events from community-based sensors.2011 10th International Conference on IEEE: Information Processing in Sensor Networks (IPSN), 13-24.

FEKETE, S., DIEDERICHS, M. \& LATO, M., 2010: Geotechnical and operational application for 3-dimensional laser scanning in drill and blast tunnels.Tunnelling and Underground Space Technol. 25: 614-628.

GARY, M., FAIRFIELD, N., STONE W.C., WETTERGREEN, D. \& KANTOR, G., 2008: 3D mapping and characterization of Sistema Zacatón from DEPTHX. "Proceedings of KARST08: 11th Sinkhole Conference ASCE, 2008: 1-11.

GÁZQUEZ, F., RULL, F., CALAFORRA J. M., SANZ A. \& MEDINA J., 2014: In situ raman spectroscopy of cave minerals: an analytical tool compatible with the conservation of subterranean heritage.- 11th GeoRaman International Conference, June 15-19, 2014, St. Louis, Missourri, USA, 5038.

GIM INTERNATIONAL, 2013: 'World's Smallest' Commercial Unmanned Aerial Lidar Platform.- http://www.gim-international.com/news/remote_sensing/lidar/ id7563-worlds_smallest_commercial_ unmanned_aerial_lidar_platform.html [Consulta: 20 de Junio de 2014].

GIM INTERNATIONAL, 2014: Debut for GPSless UAV Lidar Surveying and Mapping 
System.- http://www.gim-international. com/news/mapping/uas/id7926-debut for_gpsless_uav_lidar_surveying_and_ mapping_system.httml [Consulta: 20 de Junio del 2014].

GÓMEZ, C., 2014: Sensores en el smartphone para hacer un mapa de la contaminación.http://www.tendencias21.net/Sensores-enel-smartphone-para-hacer-un-mapa-de-lacontaminacion_a34140.html [Consulta: 20 de Junio de 2014].

GROTZINGER, J. P., CRISP, J., VASAVADA, A. R., ANDERSON, R. C., BAKER, C. J., BARRY, R., WIENS, R. C. BLAKE, D F., CONRAD P., EDGETT K. S., FERDOWSKI, B., GELLERT, R.,. GILBERT, J. B., GOLOMBEK M., GÓMEZ-ELVIRA, J., HASSLER, D. M., JANDURA, L., LITVAK, M., MAHAFFY P., MAKI, J., MEYER, M., MALIN, M. C., MITROFANOV, I., SIMMONDS, J. J., VANIMAN, D., WELCH, R. V. \& WIENS R. C., 2012: Mars Science Laboratory mission and science investigation.- Space Science Rev. 170(1-4): 5-56.

LATO, M., HUTCHINSON, D.J. \& DIEDERICHS, M, BALL, D. \& HARRAP, R., 2009: Engineered monitoring of rockfall hazards along transportation corridors: using mobile terrestrial LIDAR.- Nat Hazards Earth Syst. Sci. 9: 935-946.

LATO, M., HUTCHINSON, D.J. \& DIEDERICHS, M, 2010: Geomechanical feature extraction and analyses using LIDAR data: Iron ores company of Canada.- CIM Bull. 102(1119): 1-12.
LATO, M. J., HUTCHINSON, D. J., GAUTHIER, D., EDWARDS, T., AND ONDERCIN, M., 2014: Comparison of ALS, TLS and terrestrial photogrammetry for mapping differential slope change in mountainous terrain. Canadian Geotechnical J. 10, doi: 10.1139/cgj-2014-0051

MASON, D. C., HORRITT, M. S., HUNTER, N. M., \& BATES, P. D. 2007: Use of fused airborne scanning laser altimetry and digital map data for urban flood modelling.Hydrological Processes, 21(11): 14361447.

NASA, 2013: NASA's IceBridge Mission Contributes to new Map of Antartica.http://www.nasa.gov/topics/earth/ features/antarctic-map.html [Consulta: 03 de Julio de 2014].

NATIONAL GEOGRAPHIC, 2014: China's supercaves: Tour the Miao Room's 3-D model created from the laser scan.- http:// www.nationalgeographic.com/china-caves/supercaves/ [Consulta: 30 de Junio de 2014].

NDIAYE, M. \& DIAGNE, A., 2014: Geomagnetic Investigation Method Using Iphone ${ }^{\circledR}$ Integrated Magnetic Sensor.- Int. J. Geosci. 5: $1-4$.

OSKIN, B., 2013: 3D-printed fossils\& rocks could transform geology.- http://www.foxnews.com/science/2013/11/11/3d-printedfossils-rocks-could-transform-geology/ [Consulta: 03 de Julio de 2014].

PANASONIC, 2014: Toughpad FZ-G1.http://www.panasonic.com/business/ 
toughpad/us/durable-fz-g1-tabletphotos.asp [Consulta: 30 de Junio de 2014].

TORRES-SALINAS, D., 2011: K.1. Aplicaciones de los smartphones y la web móvil en la ciencia y la investigación.- http://digibug.ugr.es/ bitstream/10481/20219/1/305-308-K1Aplics-smartphones-ciencia-Torres Salinas-Cetto.pdf [Consulta: 27 de Junio de 2014].
SCHECK J. \& CHAO L., 2009: En plena recesión, las mini laptops baratas ganan atractivo. The Wall Street Journal.- http://live. wsj.com/article/SB124035838677941173. html?dsk=y\&mg=reno64-wsj\&url=http://online.wsj.com/article/SB124035838677941173. html [Consulta: 29 de junio de 2014].

WENG, Y.-H., SUN, F.-S., AND GRIGSBY, J. D., 2012, GeoTools: An android phone application in geology.- Computers \& Geosciences, 44: 24-30. 